\title{
Tumor-associated macrophages mediate immune suppression by secreting PD-L1+ exosomes in epithelial ovarian cancer
}

\section{Xingchen Zhou}

Shanghai Jiaotong University School of Medicine Xinhua Hospital

\section{Xiaoli Wu}

Shanghai first maternity and infant hospital

Xiaoduan Li

Shanghai first maternity and infant hospital

\section{Yi Zhang ( $\nabla$ zhangyixinhua@126.com )}

Shanghai Jiaotong University School of Medicine Xinhua Hospital https://orcid.org/0000-0003-12351257

\section{Xipeng Wang}

Shanghai Jiaotong University School of Medicine Xinhua Hospital

\section{Research article}

Keywords: Epithelial ovarian cancer, tumor-associated macrophages, exosomes, programmed cell deathligand $1, T$ cell apoptosis

Posted Date: June 20th, 2019

DOI: https://doi.org/10.21203/rs.2.10557/v1

License: (c) (i) This work is licensed under a Creative Commons Attribution 4.0 International License. Read Full License 


\section{Abstract}

Purpose: To study the role of programmed death-1/programmed death-ligand 1 (PD-1/PD-L1) signaling pathway in patients with epithelial ovarian cancer (EOC). Methods: A total of $10 \mathrm{EOC}$ specimens and 10 benign ovarian tumor were obtained from surgery and the pathological type. We used the methods of immunofluorescence confocal microscopy, western blot, MTT assay, apoptosis detection and co-culture to verify the aim of the research. Results: In the present study, it was validated that the number of PD-L1+ tumor-associated macrophages (TAMs) per field was significantly increased in EOC tissues compared with benign ovarian tumor tissues. Furthermore, it was demonstrated that PD-L1 was expressed on the membrane of TAM-derived exosomes, which may inhibit the proliferation and induce the apoptosis of T cells by activating the caspase 3 signaling pathway. The analysis of the supernatant of T cells cocultured with TAM drived exosome revealed that the levels of pro-inflammatory cytokines and tumor necrosis factor a decreased compared with those T cells co-cultured with monocyte drived exosome. However, the expression of the immuno-suppressive cytokine, interleukin 10 and markers of T cell exhaustion (the inhibitory molecule lymphocyte activated gene-3, T-cell immunoglobulin and mucin domain-containing protein-3 and PD-1) increased. Conclusions: The present study demonstrated that the M2-derived exosomes regulate immune suppression in the EOC microenvironment. The findings of the present study provide a theoretical basis for future target therapy on exosomes from immune cells to treat EOC.

\section{Background}

Epithelial ovarian cancer (EOC) is the most common cause of gynecologic cancer-associated mortality with $>2,000,000$ novel cases per year worldwide (1). Debulking surgery and platinum-based chemotherapy are the principal treatments(2). However, the initial response rate varies between 40 and $80 \%$. Furthermore, the majority of patients who initially respond to chemotherapy eventually develop chemo-resistance (2). Therapeutic strategies involving immune modulators have attracted increasing attention due to promising results from clinical trials $(3,4)$. Many studies focus on the therapeutic blockade of inhibitory signals primarily through programmed death-1 (PD-1) and its ligand programmed death-ligand 1 (PD-L1/B7-H1).

PD-1 is expressed by activated T cells and triggers an inhibitory signal via the Src homology 2 domaincontaining phosphatase-1 (5,6). PD-1 has two known ligands, PD-L1 $(7,8)$ and PD-L2 $(9,10)$. A number of studies have demonstrated that PD-L1 is selectively expressed on a number of tumor cells and also other cells within the tumor microenvironment in response to inflammatory stimuli $(11,12)$. In addition, PD-L1 is upregulated in solid tumors, therefore it is able to inhibit cytokine production and the cytolytic activity of PD $-1^{+}(C D) 4^{+}$and $C D 8^{+}$T cells in the tumor microenvironment $(11,13,14)$. In 2012 , in a study involving an antibody that specifically binds to PD-L1, Brahmer et al (9) demonstrated that $12-41 \%$ of 207 patients with advanced cancer and who received antibody treatment resulted in a prolonged stabilization of disease in non-small-cell lung cancer (NSCLC), melanoma and renal-cell cancer. However, the efficacy of the antibody was not sufficient for ovarian cancer (9). 
An interesting question is the difference between the efficacy of the antibody between EOC and other tumors. A previous study supported the existence of metastasis-supportive microenvironments, termed pre-metastatic niches, which are potential metastatic sites that allow the colonization and growth of disseminated tumor cells (15). Furthermore, our previous study revealed that the peritoneum of patients with EOC contained a large number of immune cells, $70 \%$ of which were macrophages (16). These macrophages, termed tumor associated-macrophages (TAMs), serve an important function in the regulation of $\mathrm{T}$ cell differentiation and function in the tumor microenvironment by delivering microRNAs (miRNAs) through exosomes (unpublished data).

Exosomes are vesicles of endocytic origin, which range between 30 and $100 \mathrm{~nm}$ in size (17-19), and may mediate the intercellular communication by horizontal transfer of information via their cargo. The cargo includes proteins on the membrane, DNA, mRNA and miRNAs (20-23). Exosomes may arise from numerous cell types, but the biological functions of exosomes remain unknown, as they serve complex and diverse functions in immunobiology. Previous studies demonstrated that exosomes secreted by dendritic cells may stimulate the immune system by antigen presentation, while the tumor-associated exosomes may either promote or inhibit tumor immunity, depending on the pathophysiological context (24-26). Herbst et al (12) indicated that PD-L1-positive tumor-infiltrating immune cells (macrophages, dendritic cells and T cells) were more common compared with PD-L1-positive tumor cells. Since the exosomes secreted by TAMs exhibit specific markers from the TAMs, it was hypothesized in the present study that the PD-1+ TAM-exosomes may regulate $\mathrm{T}$ cell dysfunction and apoptosis in the tumor microenvironment and participate in the formation of pre-metastatic niches, therefore contributing to immune evasion and metastasis of EOC cells. In the present study, it was demonstrated that exosomes that were released from TAMs express increased levels of PD-L1 which inhibit the proliferation and induce the apoptosis of $T$ cells by activating the caspase 3 signaling pathway, thus regulating $T$ cell dysfunction in the EOC microenvironment.

\section{Methods}

Preparation of clinical tissues. A total of 10 EOC specimens (mean age: $47.8 \pm 4.0$ (25-62) years old) and 10 benign ovarian tumor (mean age: $49.8 \pm 5.4$ (25-66) years old) specimens were obtained from the Shanghai First Maternity and Infant Hospital, Tongji University (Shanghai, China) from January 2012 to December 2014. The benign/tumor specimens were obtained from surgery and the pathological type was confirmed by two pathologists. Inclusion criteria: Epithelial ovarian cancer, none of the patients with cancer received any chemotherapy prior to the present study. Exclusion criteria: those who have received any chemotherapy prior to the present study or don't want to participate in this study. The objectives and implications of the results were explained, and institutionally approved written informed consent was obtained from each participant. The study protocol was approved by the Institutional Review Board of Shanghai First Maternity and Infant Hospital (Shanghai, China). 
Immunofluorescence confocal microscopy. The detection of PD-L $1^{+} \mathrm{TAM}$ cells (PD-L $1^{+} \mathrm{CD} 68^{+}$) in benign ovarian tumor and EOC tissues was performed using anti-PD-L1 (ab210931, Abcam, Cambridge, Cambridge, UK5ug/ml) and anti-CD68 (MAB20401, R\&D Systems, Minneapolis, MN, USA, 25ug/ml). Alexa Fluor 488-conjugated goat anti-mouse antibody (104546, Jackson, Lancaster, PA, USA, 1:200) was used for the detection of PD-L1 and Cy3-conjugated goat anti-rat antibody (99002, Jackson,1:200) was used for the detection of CD68 . cell nuclei were counterstained with DAPI (D9542, Sigma, St Louis, MO, USA). The images were captured using a laser scanning confocal microscope (Zeiss LSM 510; Zeiss GmbH, Jena, Germany). Quantitative analysis was performed on five random fields/tumor samples by counting the number of cells (magnification, $\mathrm{x} 400$ ).

M2 macrophage cell model and exosome isolation. The M2 macrophages were induced from the Thp-1 cell line (Cell bank of Chinese Academy of Sciences, Shanghai, China), using PMA (P1585-1MG, SigmaAldrich, St Louis, MO, USA, 50 ng/ml) and interleukin (IL-)4 (204-IL-010, R\&D, Minneapolis,USA,20 ng/ml) for $24 \mathrm{~h}$ with a density of $10^{6}$ /plate in RPMI1640 (Invitrogen, CA, USA)+10\%FBS (Invitrogen, CA, USA), $37^{\circ} \mathrm{C}$. In order to isolate the block mass of exosomes(exo-macs), Thp-1 cells (M0)/M2 macrophages were cultured in RPMI- 1640 for $24 \mathrm{~h} \otimes 37^{\circ} \mathrm{C} \rrbracket$. The Thp- 1 derived M2 cells were pre-stimulated into M2 macrophages using PMA and IL-4 as aforementioned for $24 \mathrm{~h} \varangle 37^{\circ} \mathrm{C} \nabla$, and subsequently the culture medium was changed to RPMI- $1640 \otimes 37^{\circ} \mathrm{C} \rrbracket$. In order to isolate exosomes, the supernatants were centrifuged twice at 1,000 $\times \mathrm{g}$ for $10 \mathrm{~min}$ and at 3,000 $\mathrm{g}$ for $30 \mathrm{~min}$ to deplete the cell or fragments under $4^{\circ} \mathrm{C}$. Subsequently, the Total Exosome Isolation kit (Life technology) was added overnight under 4 ${ }^{\circ} \mathrm{C}$. Subsequently, the exosomes were centrifuged $(10,000 \times g$ for $1 \mathrm{~h})$ under $4{ }^{\circ} \mathrm{C}$, re-suspended in PBS and stored at $-80^{\circ} \mathrm{C}$. The size and purity of the exosomes were validated using an Transmission electron microscope. The concentration of exosomes was determined using theBCA Protein Assay Kit (Pierce Biotechnology, USA).

Detection of PD-L1 on MO/M2 exosomes. The exosomes released from the M0/M2 macrophages were isolated and lysed using the Total Protein Lysis buffer (as manufacturer's instruction). The detection of PD-L1 was performed using an anti-PD-L1 antibody (ab58810, Abcam, Cambridge, UK) by western blot analysis. CD63 was used as a control reference.

Proliferation of Jurkat T cells. The proliferation of Jurkat T cells was observed using an MTT assay. The MTT reagent and Jurkat T cells were donated by the Central Lab of Shanghai First Maternity and Infant Hospital, Tongji University (Shanghai, China). In brief, the Jurkat T cells were cultured in 96 well-plates (3,000 cells/well) with $200 \mu \mathrm{l}$ RPMI-1650, supplemented with $2 \%$ FBS $\$ Invitrogen, CA, USA囚, for $24 \mathrm{~h}$. Subsequently, M0/M2-exosomes $(50 \mathrm{ng} / \mathrm{ml}$ ) were added and co-cultured for 1,2 and 3 days. The control group was treated with PBS alone. Subsequently, $20 \mu \mathrm{l}$ MTS Solution Reagent (Promega Biosciences, CA, 
USA) to each well and incubated for 2 hours at $37^{\circ} \mathrm{C}, 5 \% \mathrm{CO} 2$ atmosphere. The absorbance was recorded at $490 \mathrm{~nm}$ using a 96-well plate reader. and calculated using the standard curve performed on the first day.

Detection of apoptosis of Jurkat T cells following treatment with MO/M2-exosomes. Jurkat T cells were cultured in 6-well plates ( $1 \times 10^{6}$ cells/well) with RPMI- 1640 , supplemented with $10 \%$ FBS at $37^{\circ} \mathrm{C}, 5 \% \mathrm{CO} 2$ atmosphere. Subsequently, M0/M2-exosomes $(50 \mathrm{ng} / \mathrm{ml})$ were added and cultured for 3 days. The cells were collected and stained using the annexin $\mathrm{V}$ Apoptosis detection kit fluorescein isothiocyanate (eBioscience; Thermo Fisher Scientific, Inc., Waltham, MA, USA) according to the manufacturer's protocol. Flow cytometric analysis was performed using a fluorescence activated cell sorter (FACS) Calibur cytometer囚and data was analyzed with CellQuest software.

Detection of IL-2, IL-4, IL-6, IL-8, IL-10 and tumor necrosis factor (TNF-) a in the supernatants of Jurkat $T$ cells. The supernatants were selected following co-culture of T cells and exosomes for 3 days at $37^{\circ} \mathrm{C}, 5 \%$ $\mathrm{CO} 2$ atmosphere. The cytokines were determined using ProcartaPle ${ }^{\circledR}$ Multiplex Immunoassays (eBioscience; Thermo Fisher Scientific, Inc.) following the manufacturer's protocol.

Detection of the PD-1, lymphocyte activated gene (LAG)-3 and T-cell immunoglobulin and mucin domaincontaining protein (TIM)-3 in Jurkat T cells following treatment with MO/M2-exosomes. Jurkat T cells were cultured in 6-well plates ( $1 \times 10^{6}$ cells/well) RPMI- 1640 supplemented with $10 \%$ FBS. M0/M2exosome $(50 \mathrm{ng} / \mathrm{ml})$ was added and cultured for 3 days. Subsequently, the cells were collected and incubated with PE conjugated anti-PD-1(12-2799-41, eBioscience, Thermo Fisher Scientific, Inc.), APC conjugated anti-LAG-3 (17-2239-41, eBioscience, Thermo Fisher Scientific, Inc.) and FITC conjugated anti-TIM-3 antibodies (11-3109-41, eBioscience; Thermo Fisher Scientific, Inc.) for $30 \mathrm{~min}$ at $4^{\circ} \mathrm{C}$, protected from light. Flow cytometric analysis was performed using a FACS Calibur cytometer, and data was analyzed with CellQuest software.

Assessment of mRNA expression. Following the treatment of Jurkat T cells with $\mathrm{M} 0$ or $\mathrm{M} 2$ exosomes for 3 days, the cells were collected. The total RNA in T cells was isolated using TRIzol (Invitrogen; Thermo Fisher Scientific, Inc.) and reverse transcribed into cDNA using the miScript II RT kit (catalog no. 218161; Qiagen, Inc., Valencia, CA, USA). The templates were subjected to detection by polymerase chain reaction (PCR) using the miScript SYBR Green PCR kit (catalog no. 218073; Qiagen, Inc.). For q-PCR analysis, 500 ng of total RNA was reversed transcribed to CDNA, and amplified by PCR cycling conditions: $5 \mathrm{~s}$ at $95^{\circ} \mathrm{C}$ and $30 \mathrm{~s}$ at $60^{\circ} \mathrm{C}$ for 40 cycles. Differences in gene expression were determined by the $2 \Delta \Delta \mathrm{CT}$ method ( $\beta$ - 
actin was used for calibration). The following primer oligonucleotide sequences were used: LAG-3 forward, 5'-GCGGGGACTTCTCGCTATG-3' and reverse, 5'-GGCTCTGAGAGATCCTGGGG-3'; TIM-3 forward, 5'-CTGCTGCTACTACTTACAAGGTC-3' and reverse, 5'-GCAGGGCAGATAGGCATTCT-3'; PD-1 forward, 5'CCAGGATGGTTCTTAGACTCCC-3' and reverse, 5'-TTTAGCACGAAGCTCTCCGAT-3'.

Detection of active caspase 3 and total caspase 3 in co-cultured T cells. Following 3 days of co-cultured with M0/M2-exosome, the Jurkat T cells were collected and lysed using the Total Protein Lysis buffer (C500001-0010, Sangon Biotech, Shanghai) and concentration of protein was quantified using a BCA Protein Assay Kit (Pierce Biotechnology, USA). 20ug/lane protein was loaded in a $10 \%$ gel and then transferred with PVDF membrane. The membrane was blocked with BSA (HZB0148, sigma, USA) under room temperature for 1 hour. The primary antibody was incubated under $4^{\circ} \mathrm{C}$ overnight. The detection of the levels of active caspase 3 and the total levels of caspase 3 was performed using the rabbit anti human-anti-active-caspase 3 antibody $(\mathrm{ab} 2302$, abcam, $1 \mathrm{ug} / \mathrm{ml})$ and rabbit anti human-anti-caspase 3 antibody (ab32351, Abcam, 1:5000) The second antibody was using the goat anti-rabbit IgG(HRP) antibody (ab6721, abcam) and incubated under room temperature for 1 hour. The valuation of the protein concentration was using the Pierce ${ }^{\mathrm{TM}}$ ECL Western Blotting Substrate $\left(32106\right.$, Thermo Scientific ${ }^{\mathrm{TM}}$, USA).

Statistical analysis. Statistical analyses were performed using the SPSS software (version 19.0; IBM Corp., Armonk, NY, USA). The data are expressed as the mean \pm standard deviation. The Mann-Whitney test, univariate analysis of variance with post-hoc LSD test and Kruskal-Wallis test were used to determine the P-values. The continuous variables in the figures are presented as the mean \pm standard error of the mean. $\mathrm{P}<0.05$ was considered to indicate a statistically significant difference.

\section{Results}

Number of PD-L $1^{+}$TAMs is significantly increased in EOC tissues. The frequency of PD-L1 ${ }^{+}$TAM (PD$\mathrm{L} 1^{+} \mathrm{CD} 68^{+}$) cells in $10 \mathrm{EOC}$ specimens and 10 benign ovarian tumor specimens were evaluated using immunofluorescence confocal microscopy (Fig. 1A). The number of PD-L1+ TAMs per field was significantly increased in EOC tissues compared with benign ovarian tumor tissues (17.80 $\pm 1.8 \mathrm{vs}$. 4.60 \pm 0.5 , Mann-Whitney test, $P=0.01$ ) (Fig. 1B). This result was consistent with Herbst et al (12).

$P D-L 1$ expression is significantly increased in M2-exosomes. The expression of PD-L1 was increased in the exosomes that were released by M2 macrophages. A previous study by the authors revealed that the exosomes released by M2 macrophages might regulate the differentiation of T cells via miRNAs (data in submission). Since exosomes carry specific proteins from the host cell, exosomes from M2 macrophages were purified and examined using an electron microscope (Fig. 2A). Subsequently, PD-L1 expression in 
the exosomes was determined. The expression of PD-L1 was significantly increased in the M2-exosome group compared with the control group (Mann-Whitney test, $\mathrm{P}=0.002$ ) (Fig. 2B and C).

Exosomes released from $\mathrm{M} 2$ macrophage are able to reduce the proliferation of Jurkat $T$ cells. The exosomes from M2 macrophages were collected and co-cultured with Jurkat T cells. Following co-culture with M2 exosomes for 2 days, the proliferation of Jurkat T cells was significantly decreased compared with the M0-exo group and the control group (M2: $5348.70 \pm 41.13$ cells/well vs. control: $7112.26 \pm 441.09$ cells/well; M0: $6146.35 \pm 237.27$ cells/well; $\mathrm{M} 2$ vs control: $\mathrm{P}=0.005, \mathrm{M} 2$ vs $\mathrm{M0}$ : $\mathrm{P}=0.1$. ANOVA). A similar result was observed after 3 days of co-culture (control, 10718.54 \pm 346.1 cells/well; M0, 10079.23 \pm 137.32 cells/well; M2, 7245.52 \pm 80.32 cells/well; M2 vs control: $P<0.001$, M2 vs M0: $P<0.001$. ANOVA) (Fig. 3).

Exosome released from M2 macrophages regulate the apoptosis of Jurkat T cells. Mediation of T cell apoptosis by PD-L1 is a novel mechanism for tumor immune escape $(4,5)$. In the present study, the apoptosis of Jurkat T cells was determined following co-culture with M2-exosome/M0 (Thp-1)-exosome for 3 days. The exosome released from M2 macrophages (early apoptosis, $36.43 \pm 1.44 \%$; late apoptosis, $22.13 \pm 0.49 \%$;) significantly induced the apoptosis of Jurkat T cells compared with the M0-exosome (early apoptosis, $9.52 \pm 4.03 \%$; late apoptosis, $6.82 \pm 2.74 \%$ ) and the control group (early apoptosis, $13.06 \pm 2.69 \%$; late apoptosis, $4.62 \pm 0.15 \%$ ) (early apoptosis, $L S D, M 2$ vs control: $P=0.001 ; M 2$ vs $M 0: P=0.001$; late apptosis, Tamhane: $M 2$ vs control: $P=0.001 ; M 2$ vs $M 0: P=0.08$ ) (Fig. $4 A-C$ )

Exosomes released from $M 2$ macrophage upregulate the secretion of IL-10 from Jurkat $T$ cells. The aim of the next part of the study was to determine if the M2-exosome affect the function of Jurkat T cells. The ProcartaPlex ${ }^{\circledR}$ Multiplex Immunoassays were used to determine the release of IL-2, IL-4, IL-6, IL-8, IL10 and TNF-a from the supernatants of Jurkat T cells following co-culture with M2-exosome/M0exosome for 3 days. The pro-inflammatory cytokines, IL-2, IL-8, IL-4, IL-6 and TNF-a, were significantly decreased in the M2-exo group (IL-2: 2.16 \pm 2.16 pg/ml; IL-8: 0 pg/ml; IL-4: 0 pg/ml; IL-6: 0 pg/ml; TNF-a: 0 $\mathrm{pg} / \mathrm{ml}$ ) compared with the M0-exo group (IL-2: $16.48 \pm 0.56 \mathrm{pg} / \mathrm{ml} ; \mathrm{IL}-8: 1258.36 \pm 24.59 \mathrm{pg} / \mathrm{ml}$; IL-4: $16.52 \pm 0.31 \mathrm{pg} / \mathrm{ml} ; \mathrm{IL}-6: 25.69 \pm 0.62 \mathrm{pg} / \mathrm{ml}$; TNF-a: $18.58 \pm 0.94 \mathrm{pg} / \mathrm{ml})$ and the control group (IL-2: $9.35 \pm 0.50$ pg/ml; IL-8: $588.4 \pm 2.70$ pg/ml; IL-4: $34.13 \pm 1.13$ pg/ml; IL-6: 0 pg/ml; TNF-a: $10.71 \pm 0.51$ pg/ml) (IL-2: M2 vs M0: $P=0.05$, M2 vs control: $P=0.046$, Mann-Whitney; IL-8: $M 2$ vs $M 0: P=0.046, M 2$ vs control: $\mathrm{P}=0.034$, Mann-Whitney; IL-4: M2 vs M0: $\mathrm{P}=0.05$, M2 vs control: $\mathrm{P}=0.037$, Mann-Whitney; IL-6: M2 vs M0: $P=0.037$, M2 vs control: $P=1$, Mann-Whitney; TNF-a: $M 2$ vs $M 0: P<0.001, M 2$ vs control: $P<0.001, A N O V A$, (Fig. 5). Whereas, IL-10 was significantly increased in M2-exo group ( $5.57 \pm 0.38 \mathrm{pg} / \mathrm{ml}$;) compared with the other two groups ( $M 0: 3.04 \pm 0.07 \mathrm{pg} / \mathrm{ml}$; control: $0 \mathrm{pg} / \mathrm{ml}, \mathrm{M} 2$ vs control: $P=0.037, M 2$ vs $M 0: P=0.037$, Mann-Whitney) (Fig. 5E). IL-8, TNF-a, IL-4 and IL-6 were undetected in the M2-exo group. 
Markers of Jurkat T cell exhaustion are upregulated by exosomes released from M2 macrophages. The exosomes from M2 macrophages are able to induce apoptosis and upregulate the secretion of the antiinflammatory cytokine, IL-10. Therefore, in the present study, the markers of T cell exhaustion, LAG-3, TIM3 and PD-1, were determined in Jurkat T cells. The protein levels of all three markers were upregulated in the M2-exo group compared with the control (LAG-3: $38.63 \pm 5.32 \%$ vs. $2.73 \pm 0.45 \%$, ANOVA, $P=0.003$; TIM3: $7.41 \pm 0.58 \%$ vs. $1.32 \pm 0.09 \%$, ANOVA, $P<0.001$; PD-1: $21.25 \pm 3.73 \%$ vs. $2.80 \pm 0.14 \%$; ANOVA, $P=0.008$ ) (Fig. 6A). Furthermore, the levels of LAG-3 (255.08 \pm 94.22 vs. $7.45 \pm 3.23$; Mann-Whitney, $P>0.05)$, TIM-3 (104.44 \pm 4.47 vs. $2.39 \pm 1.50$; ANOVA, $P<0.001)$ and PD-1 mRNA (19.45 \pm 2.91 vs. $1.67 \pm 0.56$; ANOVA, $\mathrm{P}=0.004$ ) were upregulated in the M2-exo group compared with the control (Fig. $6 \mathrm{~B}$ ). It was also validated that M2 macrophages were able to induce apoptosis and exhaustion of Jurkat $\mathrm{T}$ cells by activating caspase $3(P=0.011$, ANOVA) (Fig. $6 \mathrm{C}$ and $\mathrm{D})$

\section{Discussion}

The PD-1/PD-L1 pathway is an important immune inhibitory signaling pathway in the tumor microenvironment $(6,27)$. An increased PD-L1 expression in the tumor microenvironment is associated with poor prognosis in patients with a number of types of cancer, including breast, ovarian, pancreatic, gastric, kidney and bladder cancer (28-33).

Multiple monoclonal antibodies to PD-L1 are under development in clinical trials. It has been demonstrated that BMS-936559 (anti-PD-L1) exhibited therapeutic efficacy in a phase I clinical trial (ClinicalTrials.gov identifier, NCT00729664) (9). In addition, a study of metastatic urothelial bladder cancer identified MPDL3280A (an engineered anti-PD-L1 Immunoglobulin G1 monoclonal antibody) to have marker activity in controlling tumor growth; the objective response rates (ORRs) were $43 \%$ for PD$\mathrm{L} 1^{+}$tumor and $11 \%$ for PD-L1 ${ }^{-}$tumors. (ClinicalTrials.gov identifier, NCT01375842) (34). Furthermore, in a phase I trial, patients with different types of cancer (melanoma, NSCLC and renal cell carcinoma) responded to MPDL3280A with an ORR of $23 \%$ total patients with NSCLC and an ORR of $85 \%$ in patients with high PD-L1 expression (NCT01375842) (12). However, ovarian cancer has not been demonstrated to respond to MPDL3280A (9). Therefore, in the present study, it was hypothesized that other types of cells, in addition to tumor cells, may express PD-L1 and contribute to tumor proliferation and metastasis. According to a previous study by the authors, the peritoneum of patients with EOC contained a high number of immune cells, $70 \%$ of which were TAM cells. In the present study, it was validated that TAM cells in the EOC tumor microenvironment expressed PD-L1, which was consistent with the results of Herbst et al (12), and that PD-L1 may be expressed on the membrane of exosomes (small vesicles ranging between 30 and $100 \mathrm{~nm}$ in size) that are released from TAM cells.

Exosomes are one of the major means of communication employed by immune cells. Exosomes contain a combination of ligands and receptors that may concurrently interact with a number of cell-surface receptors, therefore mediating the exchange of membrane and cytosolic components without cell-cell 
contact. For example, antigen-presenting cells release exosomes exhibit major histocompatibility complex (MHC) class II/antigen complexes, resulting in the activation of $T$ cells via specific $T$ cell receptor-peptide interactions $(35,36)$. Additionally, mast cell-derived exosomes may stimulate $T$ cells via the MHC class II molecules (37). A previous study in 2012 revealed that the exosomes released from the first trimester and term placenta regulates immune tolerance (6). Furthermore, it was demonstrated that dendritic cells that were exposed to tumor peptides generated exosomes that were able to induce a T-cellmediated anti-tumor immune response (26). In the present study, it was observed that the PD-L $1^{+}$ exosomes released from M2 macrophages, similar to TAM cells in the EOC microenvironment, may inhibit the proliferation of T cells and induce the apoptosis of T cells by activating the caspase 3 signaling pathway. According to Duraiswamy et al (7), it was demonstrated that the expansion of ovarian antigenspecific CD $8^{+}$tumor infiltrating lymphocytes was dependent on the amount of PD-L1 signaling by tumor cells, tumor-derived myeloid cells and T regulatory cells. The present results were partly consistent with these findings. Furthermore, the present results offer a better explanation for the low efficacy of molecular targeted therapeutic drugs specific to PD-L1. As exosomes are small and are released in a huge amount, if the PD-L1 on tumor or TAM cells is inhibited, the nomadic and diffused TAM-derived PD-L $1^{+}$exosomes may serve their function in immune suppression. The small size of the exosomes enables a diffuse spread of these vesicles, therefore forming pre-metastatic niches on the peritoneum for EOC tumor cells.

In the present study, it was demonstrated that the M2-derived exosomes were able to regulate the release of cytokines from T cells. The levels of the pro-inflammatory cytokines, IL-2, IL-8, IL4, IL 6 and TNF-a, decreased, by contrast, levels of the immuno-suppressive cytokine, IL-10, was increased compared with control and $\mathrm{M} 0$ group. These results were consistent with the study by Dong et al (38). Furthermore, the markers of T cell exhaustion, PD-1, LAG-3 and TIM-3, were upregulated in T cells. A previous study demonstrated that anti-PD-L1 monoclonal antibody might reverse $T$ cell exhaustion and restore the function of T cells (39). Thus, we can hypothesis that the accumulation of high levels of immunesuppressive cytokines may explain why PD-L1 blockade in vivo only exhibited a partial anti-tumor effect.

The results of the present study identified that TAMs mediated the dysfunction of T cells by releasing PD$\mathrm{L}^{+}$exosomes in the EOC tumor microenvironment, therefore promoting the proliferation of tumor cells and the formation of pre-metastatic niches on the peritoneum. Further investigation on the release of exosomes from immune cells may enable the identification of a novel treatment for EOC.

\section{Declarations}

\section{Funding}

The present study was supported by the National Science Foundation of China (grant no. 81372787)

\section{Availability of data and materials}

The datasets used and/or analyzed during the current study are available from the corresponding author on reasonable request. 


\section{Authors' contributions}

Dr. Zhou X have done the experiments, Dr. Zhu Q and Dr. Liu L helped to collected the clinical tissues, Dr. Zhang $Y$ analysised the data and Pro. Wang designed and wrote the article.

\section{Ethics approval and consent to participate}

The study protocol was approved by the Institutional Review Board of Shanghai First Maternity and Infant Hospital (Shanghai, China).

\section{Consent for publication}

All authors have reviewed the final version of the manuscript and approve it for submission.

\section{Competing interests}

All the authors declare no conflicts of interest.

\section{References}

1. Ferlay J, Shin HR, Bray F, Forman D, Mathers $C$ and Parkin DM: Estimates of worldwide burden of cancer in 2008: GLOBOCAN 2008. International journal of cancer. Journal international du cancer 127: 2893-2917, 2010.

2. Aletti GD, Gallenberg MM, Cliby WA, Jatoi A and Hartmann LC: Current management strategies for ovarian cancer. Mayo Clinic proceedings 82: 751-770, 2007.

3. Page DB, Postow MA, Callahan MK, Allison JP and Wolchok JD: Immune modulation in cancer with antibodies. Annual review of medicine 65: 185-202, 2014.

4. Finn OJ: Immuno-oncology: understanding the function and dysfunction of the immune system in cancer. Annals of oncology : official journal of the European Society for Medical Oncology / ESMO 23 Suppl 8: viii6-9, 2012.

5. Pardoll DM: The blockade of immune checkpoints in cancer immunotherapy. Nature reviews. Cancer 12: 252-264, 2012.

6. Keir ME, Butte MJ, Freeman GJ and Sharpe AH: PD-1 and its ligands in tolerance and immunity. Annual review of immunology 26: 677-704, 2008.

7. Harvey RD: Immunologic and Clinical Effects of Targeting PD-1 in Lung Cancer. Clinical Pharmacology \& Therapeutics 96: 214-223, 2014.

8. Naidoo J, Page DB and Wolchok JD: Immune modulation for cancer therapy. British Journal of Cancer 111: 2214-2219, 2014.

9. Brahmer JR, Tykodi SS, Chow LQ, et al.: Safety and activity of anti-PD-L1 antibody in patients with advanced cancer. The New England journal of medicine 366: 2455-2465, 2012. 
10. Hamid O, Robert C, Daud A, et al.: Safety and tumor responses with lambrolizumab (anti-PD-1) in melanoma. The New England journal of medicine 369: 134-144, 2013.

11. Wolchok JD, Kluger H, Callahan MK, et al.: Nivolumab plus ipilimumab in advanced melanoma. The New England journal of medicine 369: 122-133, 2013.

12. Herbst RS, Soria J-C, Kowanetz M, et al.: Predictive correlates of response to the anti-PD-L1 antibody MPDL3280A in cancer patients. Nature 515: 563-567, 2014.

13. Budiu RA, Elishaev E, Brozick J, et al.: Immunobiology of human mucin 1 in a preclinical ovarian tumor model. Oncogene 32: 3664-3675, 2013.

14. Lau SK, Weiss LM and Chu PG: Differential expression of MUC1, MUC2, and MUC5AC in carcinomas of various sites: an immunohistochemical study. American journal of clinical pathology 122: 61-69, 2004.

15. Sceneay J, Smyth MJ and Moller A: The pre-metastatic niche: finding common ground. Cancer metastasis reviews 32: 449-464, 2013.

16. Wang X, Deavers M, Patenia R, et al.: Monocyte/macrophage and T-cell infiltrates in peritoneum of patients with ovarian cancer or benign pelvic disease. Journal of translational medicine 4: 30, 2006.

17. Arscott WT and Camphausen KA: EGFR isoforms in exosomes as a novel method for biomarker discovery in pancreatic cancer. Biomarkers in medicine 5: 821, 2011.

18. Record M, Carayon K, Poirot M and Silvente-Poirot S: Exosomes as new vesicular lipid transporters involved in cell-cell communication and various pathophysiologies. Biochimica et biophysica acta 1841: 108-120, 2014.

19. S ELA, Mager I, Breakefield XO and Wood MJ: Extracellular vesicles: biology and emerging therapeutic opportunities. Nature reviews. Drug discovery 12: 347-357, 2013.

20. Choi DS, Kim DK, Kim YK and Gho YS: Proteomics, transcriptomics and lipidomics of exosomes and ectosomes. Proteomics 13: 1554-1571, 2013.

21. Martins VR, Dias MS and Hainaut P: Tumor-cell-derived microvesicles as carriers of molecular information in cancer. Current opinion in oncology 25: 66-75, 2013.

22. Peinado H, Lavotshkin S and Lyden D: The secreted factors responsible for pre-metastatic niche formation: old sayings and new thoughts. Seminars in cancer biology 21: 139-146, 2011.

23. Thakur BK, Zhang H, Becker A, et al.: Double-stranded DNA in exosomes: a novel biomarker in cancer detection. Cell research 24: 766-769, 2014.

24. Wolfers J, Lozier A, Raposo G, et al.: Tumor-derived exosomes are a source of shared tumor rejection antigens for CTL cross-priming. Nature medicine 7: 297-303, 2001.

25. Taylor DD and Gercel-Taylor C: Tumour-derived exosomes and their role in cancer-associated T-cell signalling defects. Br J Cancer 92: 305-311, 2005.

26. Zitvogel L, Regnault A, Lozier A, et al.: Eradication of established murine tumors using a novel cellfree vaccine: dendritic cell-derived exosomes. Nature medicine 4: 594-600, 1998. 
27. Keir ME, Francisco LM and Sharpe AH: PD-1 and its ligands in T-cell immunity. Current opinion in immunology 19: 309-314, 2007.

28. Inman BA, Sebo TJ, Frigola X, et al.: PD-L1 (B7-H1) expression by urothelial carcinoma of the bladder and BCG-induced granulomata: associations with localized stage progression. Cancer 109: 14991505, 2007.

29. Konishi J, Yamazaki K, Azuma M, Kinoshita I, Dosaka-Akita H and Nishimura M: B7-H1 expression on non-small cell lung cancer cells and its relationship with tumor-infiltrating lymphocytes and their PD1 expression. Clinical cancer research : an official journal of the American Association for Cancer Research 10: 5094-5100, 2004.

30. Nakanishi J, Wada Y, Matsumoto K, Azuma M, Kikuchi K and Ueda S: Overexpression of B7-H1 (PDL1) significantly associates with tumor grade and postoperative prognosis in human urothelial cancers. Cancer immunology, immunotherapy : CII 56: 1173-1182, 2007.

31. Nomi T, Sho M, Akahori T, et al.: Clinical significance and therapeutic potential of the programmed death-1 ligand/programmed death-1 pathway in human pancreatic cancer. Clinical cancer research : an official journal of the American Association for Cancer Research 13: 2151-2157, 2007.

32. Thompson RH, Gillett MD, Cheville JC, et al.: Costimulatory B7-H1 in renal cell carcinoma patients: Indicator of tumor aggressiveness and potential therapeutic target. Proceedings of the National Academy of Sciences of the United States of America 101: 17174-17179, 2004.

33. Wu C, Zhu Y, Jiang J, Zhao J, Zhang XG and Xu N: Immunohistochemical localization of programmed death-1 ligand-1 (PD-L1) in gastric carcinoma and its clinical significance. Acta histochemica 108: 19-24, 2006.

34. Powles T, Eder JP, Fine GD, et al.: MPDL3280A (anti-PD-L1) treatment leads to clinical activity in metastatic bladder cancer. Nature 515: 558-562, 2014.

35. Admyre C, Johansson SM, Paulie S and Gabrielsson S: Direct exosome stimulation of peripheral human T cells detected by ELISPOT. Eur J Immunol 36: 1772-1781, 2006.

36. Arnold PY and Mannie MD: Vesicles bearing MHC class II molecules mediate transfer of antigen from antigen-presenting cells to CD4+ T cells. Eur J Immunol 29: 1363-1373, 1999.

37. Vincent-Schneider $H$, Stumptner-Cuvelette P, Lankar D, et al.: Exosomes bearing HLA-DR1 molecules need dendritic cells to efficiently stimulate specific T cells. International immunology 14: 713-722, 2002.

38. Dong H, Zhu G, Tamada K and Chen L: B7-H1, a third member of the B7 family, co-stimulates T-cell proliferation and interleukin-10 secretion. Nature medicine 5: 1365-1369, 1999.

39. Barber DL, Wherry EJ, Masopust D, et al.: Restoring function in exhausted CD8 T cells during chronic viral infection. Nature 439: 682-687, 2006.

\section{Figures}




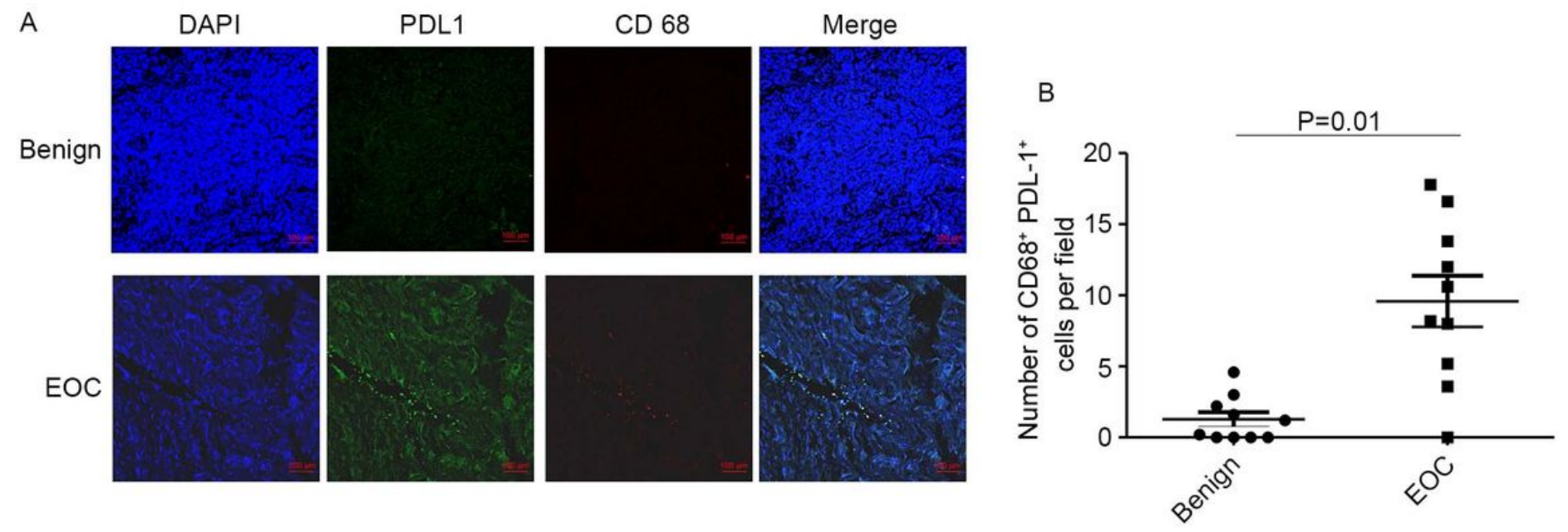

\section{Figure 1}

Distribution of PD-L1+ TAM cells in EOC tissues and benign ovarian tumor samples. (A) PD-L1+ TAM cells were stained using DAPI (blue, nuclei), PD-L1 (green), and CD68 (red) in benign ovarian tumor tissues and EOC tissues. Scale bar, $100 \mu \mathrm{m}$. (B) The number of PD-L1+ TAM cells in EOC tissues was higher compared with the benign ovarian tumor samples. PD-L1, programmed death-ligand 1; TAM, tumor-associated macrophage; EOC, epithelial ovarian cancer; $C D$, cluster of differentiation.

A

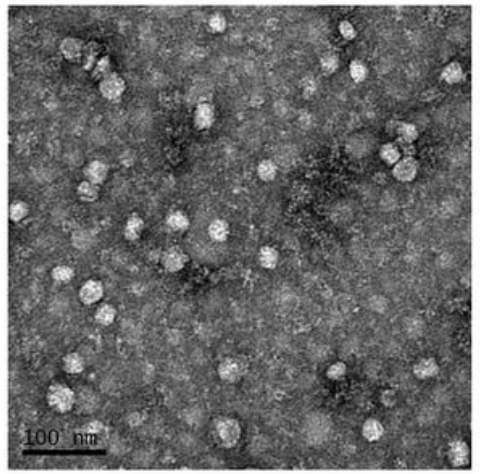

B

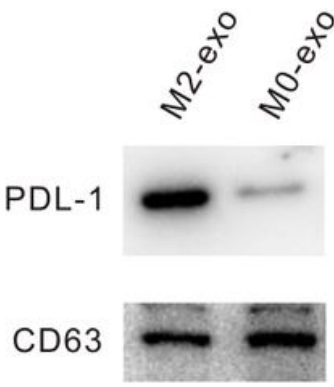

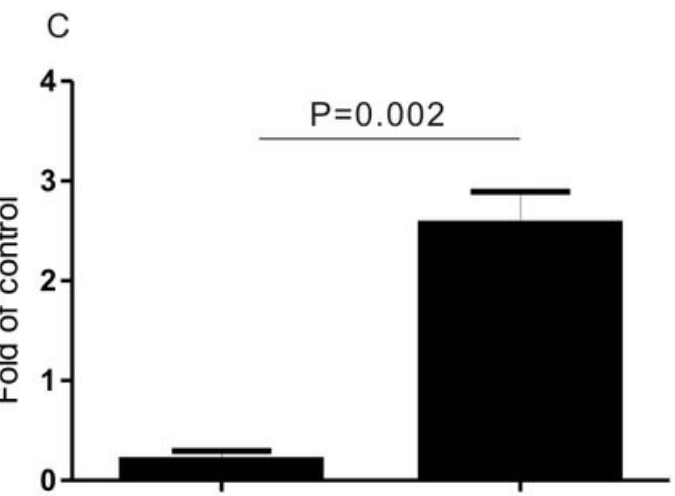

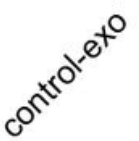

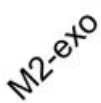

\section{Figure 2}

Expression of PD-L1 in the exosomes released by M2/M0 macrophages. (A) The size of the exosomes was determined to be 30-100 nm using an electron microscope. Scale bar, $100 \mathrm{~nm}$. (B) PD-L1 expression in the exosomes released from M2 (Thp-1 induced)/M0 (Thp-1) macrophages as determined by western blotting. CD63 was used as the control. (C) PD-L1 expression in the exosomes released by M2/M0 macrophages. Bars represents mean \pm standard error of the mean. PD-L1, programmed death-ligand 1; $\mathrm{CD}$, cluster of differentiation; Exo, exosome. 


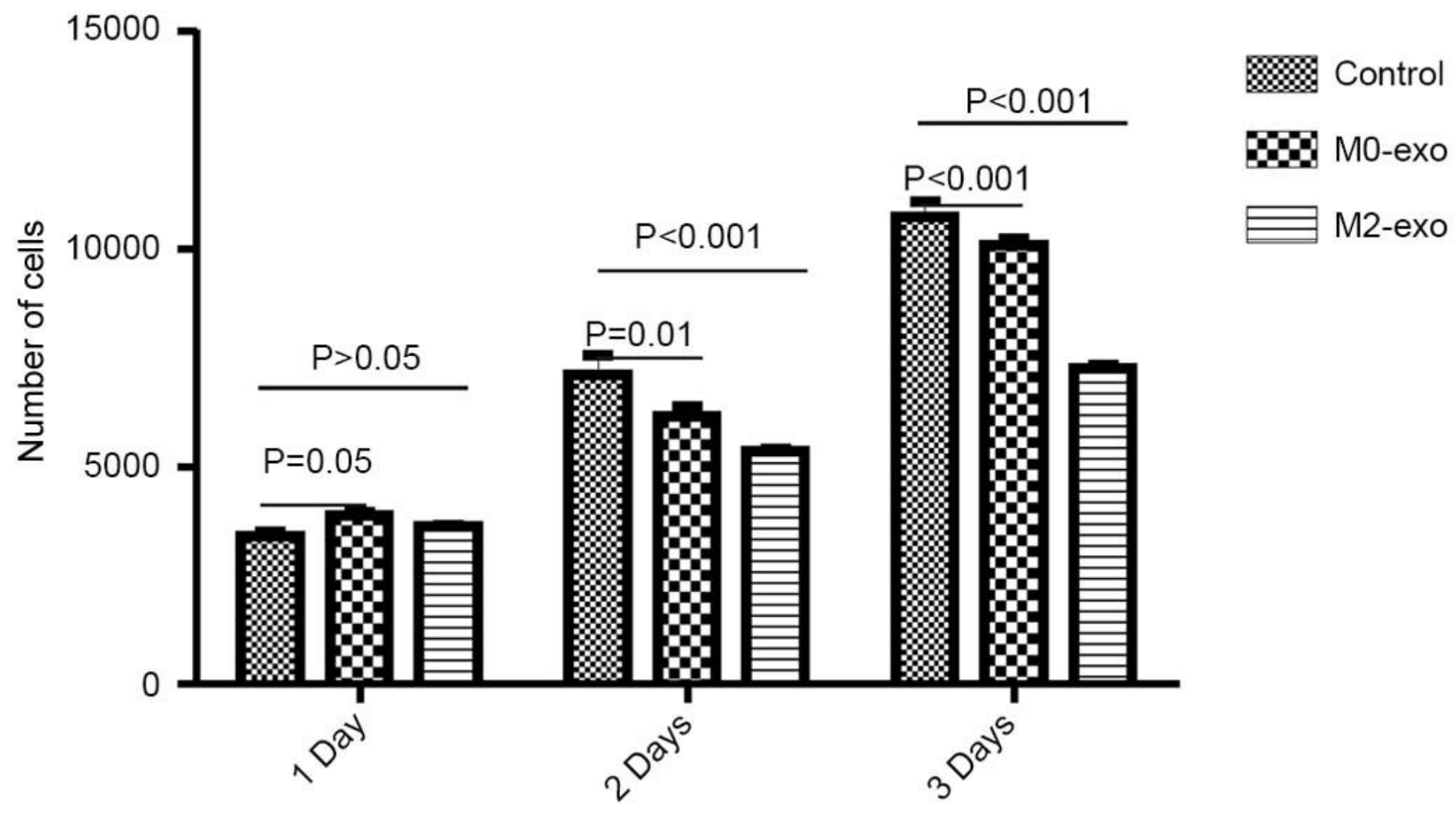

Figure 3

Exosomes released from M2 macrophages regulate the proliferation of Jurkat T cells. Jurkat cells were cultured with $\mathrm{M} 2$ or $\mathrm{M} 0$ exosomes in RPMI-1640 medium for 1, 2 and 3 days. The number of cells was calculated using an MTT assay. Exo, exosome. ${ }^{*} \mathrm{P}<0.05 ; * * \mathrm{P}<0.01$. 
A

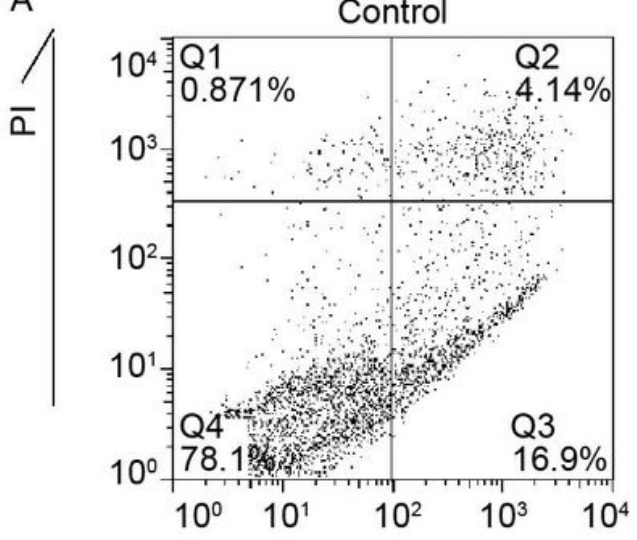

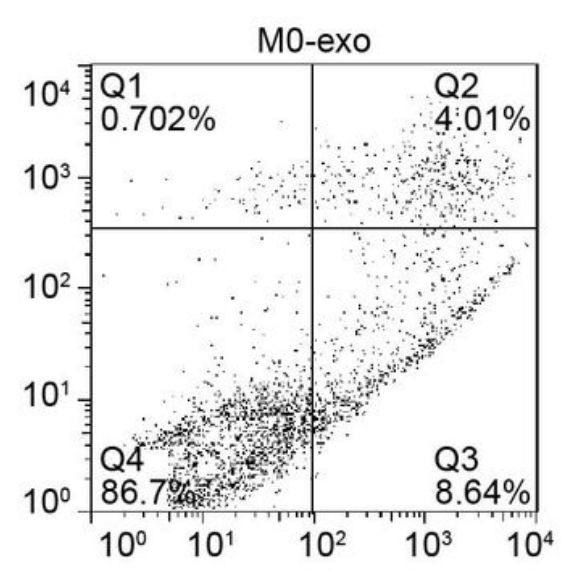

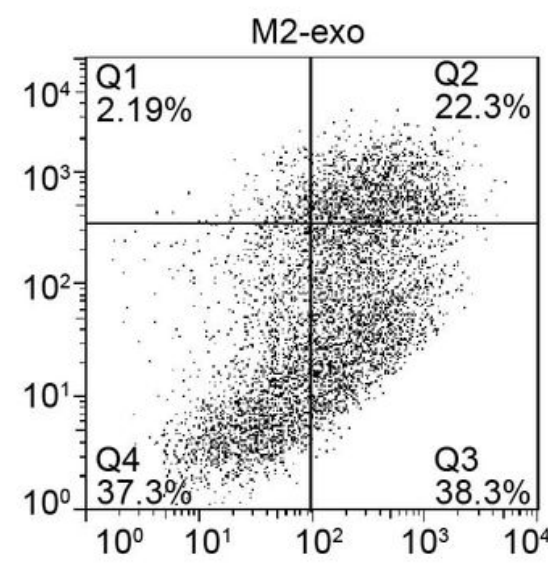

Annexin-V

B

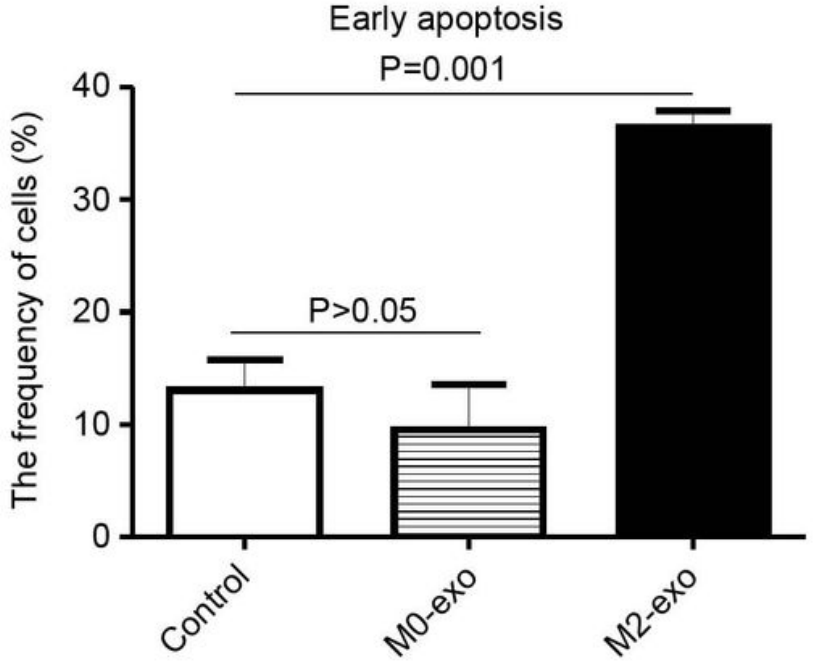

C

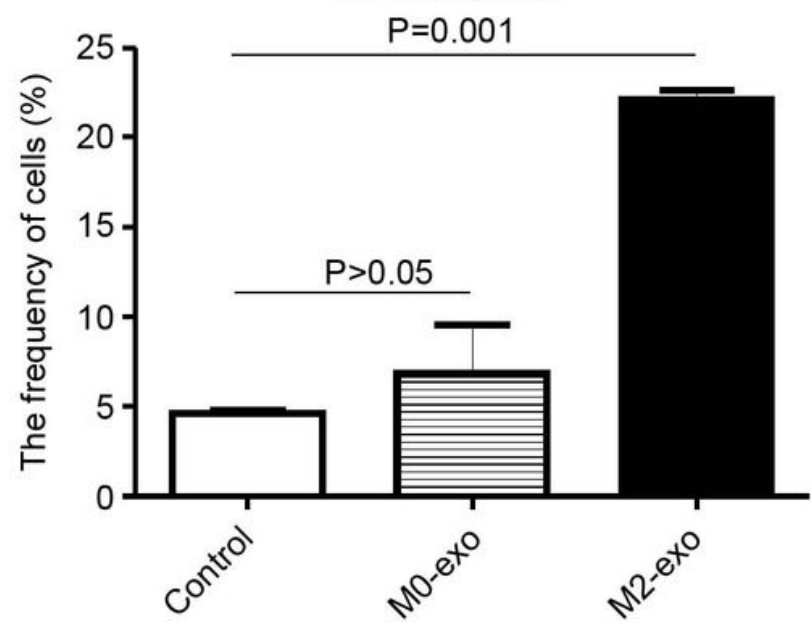

Figure 4

Exosomes released from M2 macrophage regulate the apoptosis of Jurkat T cells. (A) Jurkat T cells were cultured with M2 or M0 exosomes in RPMI-1640 medium for 3 days. The apoptosis of Jurkat T cells was analyzed using a fluorescence activated cell sorter with an annexin V apoptosis detection kit. The proportion of cells in (B) early apoptosis and (C) late apoptosis were determined. The exosomes released form M2 macrophage significantly induced the apoptosis of Jurkat T cells compared with the M0exosome and control groups. PI, propidium iodide; Exo, exosome. 
A

IL-2

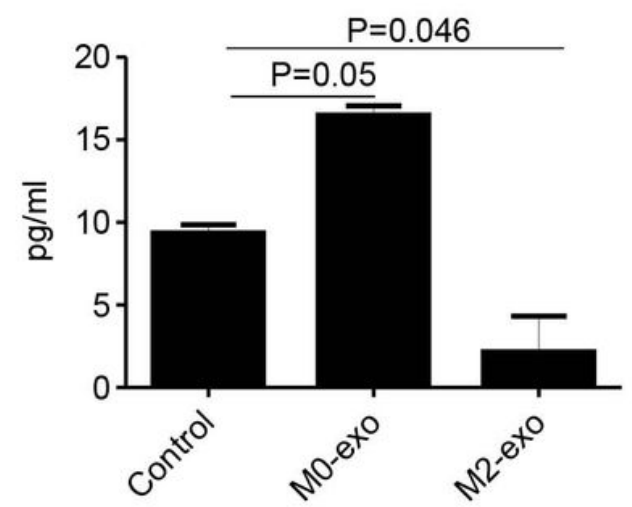

D

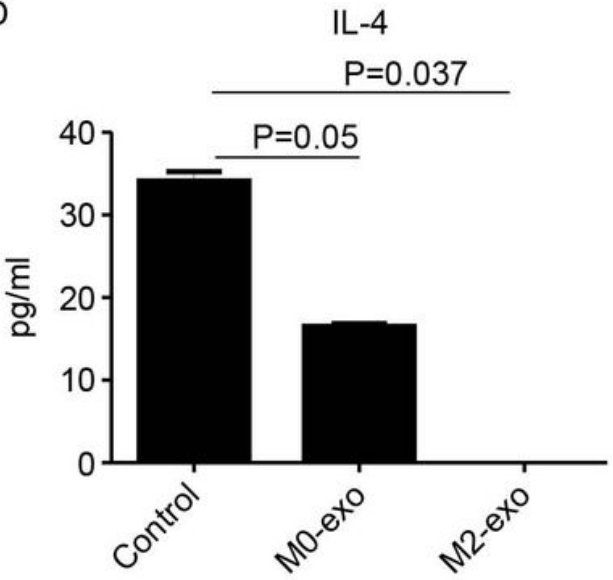

B

IL-8

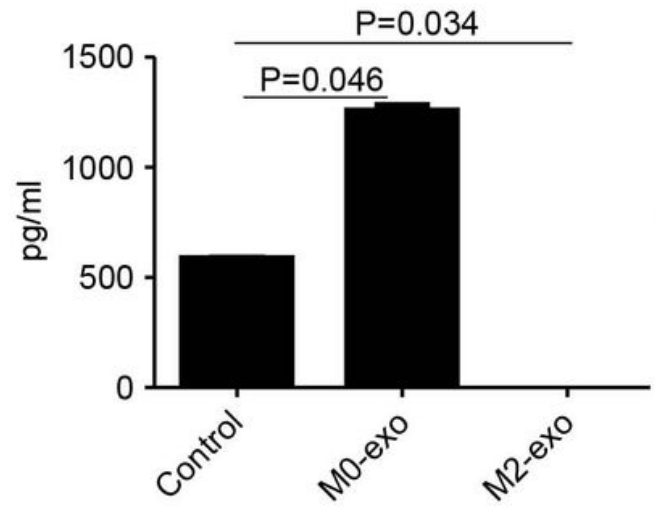

E

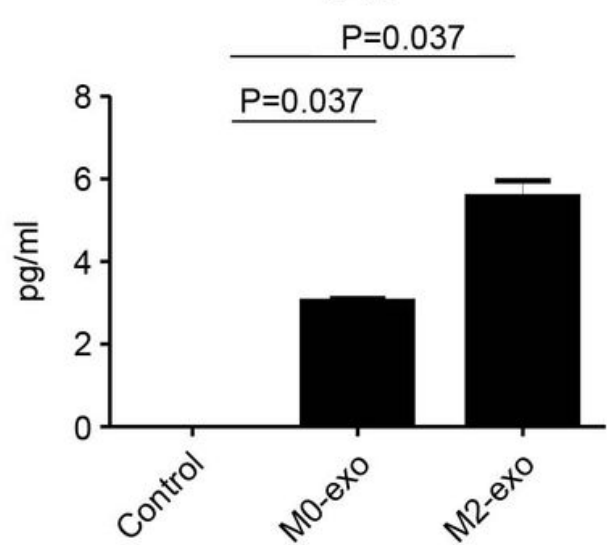

C TNF- $\alpha$

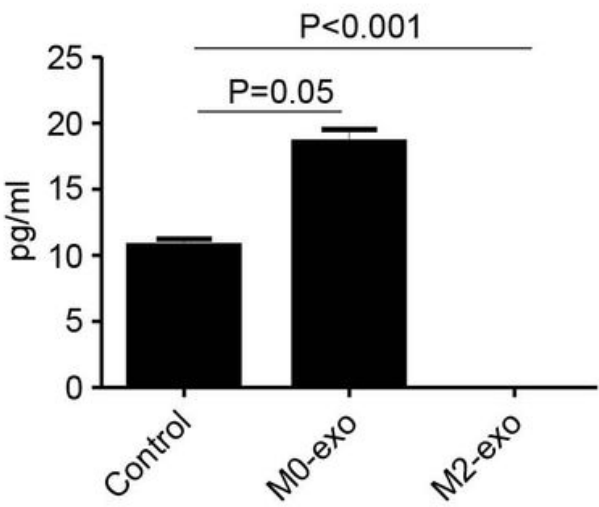

F

IL-6

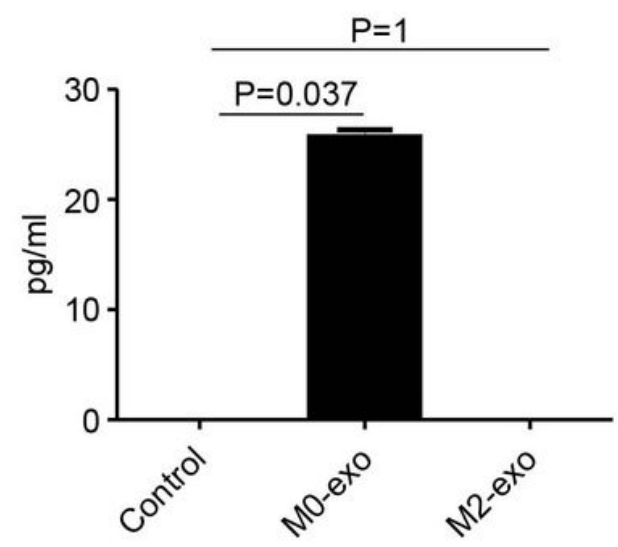

Figure 5

Detection of IL-2, $-4,-6,-8$ and -10 and TNF-a in the supernatants of Jurkat T cells. The cytokines, (A) IL-2, (B) IL-8, (C) TNF-a, (D) IL-4, (E) IL-10 and (F) IL-6, were determined using ProcartaPlex® Multiplex immunoassays according to the manufacturer's protocol. Pro-inflammatory cytokines, IL-2, IL-8, TNF-a, IL4 and IL-6, were significantly decreased in the M2-exo group, but IL-10 was significantly increased in the M2-exo group compared with the control and M0-exo groups. IL, interleukin; TNF-a, tumor necrosis factor a; Exo, exosome. ${ }^{*} \mathrm{P}<0.05 ; * * \mathrm{P}<0.01$ 
A

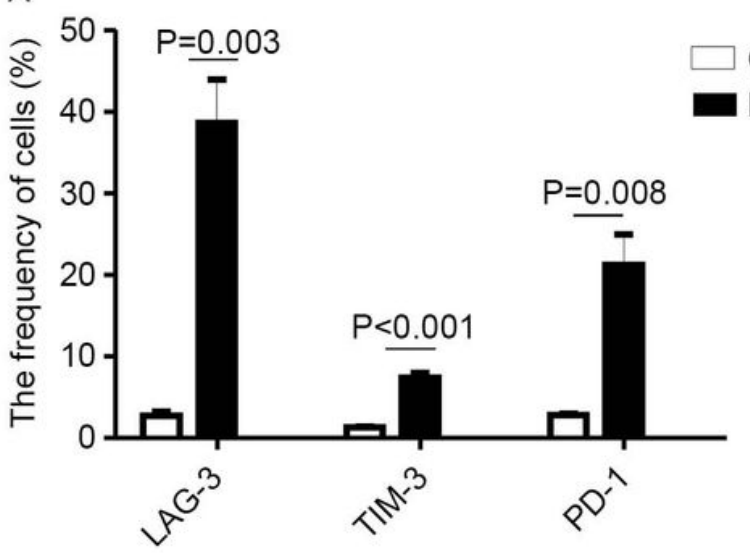

C

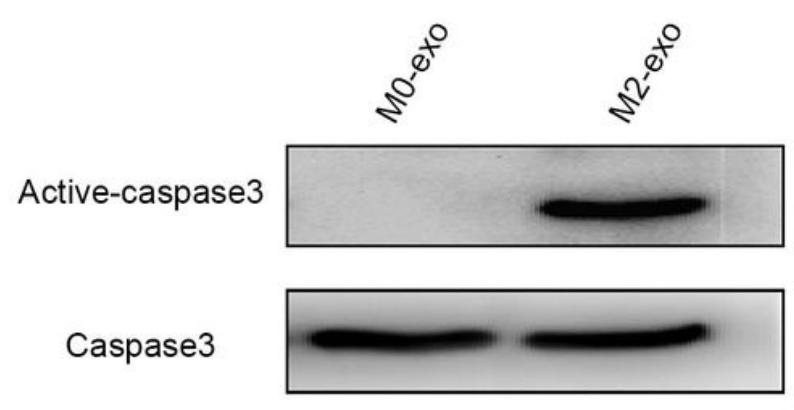

B

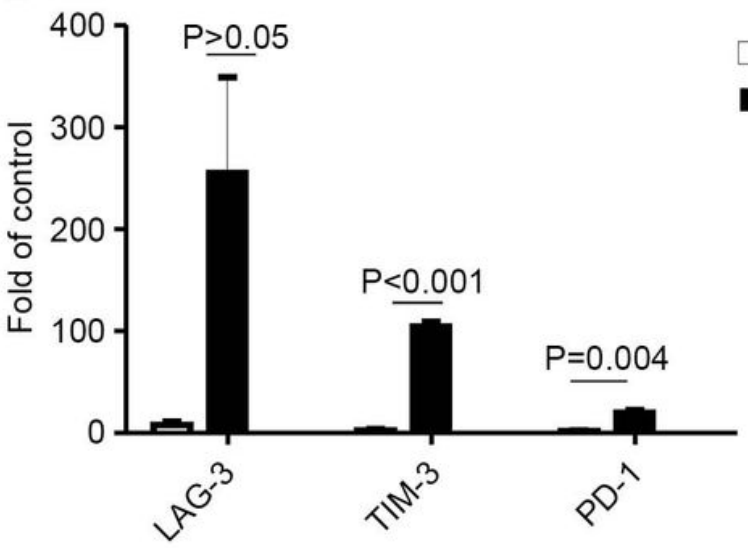

D

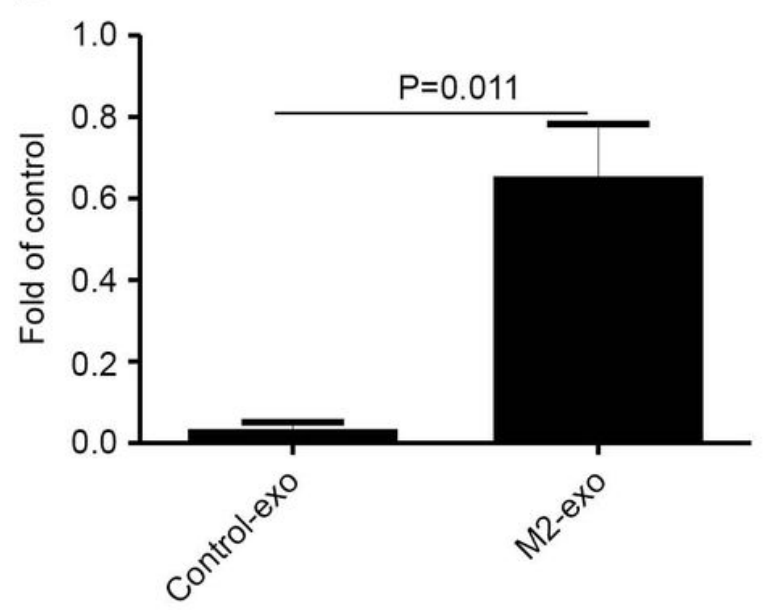

Figure 6

Regulation of markers of Jurkat T cell exhaustion by exosomes released from M2 macrophages. Jurkat T cells were cultured with $\mathrm{M} 2$ or $\mathrm{M} 0$ exosomes in RPMI-1640 medium for 3 days. (A) Following co-culture, the cells were collected and the expression of LAG-3, TIM-3 and PD-1 was determined using a fluorescence activated cell sorter. (B) The mRNA levels of LAG-3, TIM-3 and PD-1 were determined using polymerase chain reaction. (C) The expression of active-caspase 3 was determined in Jurkat T cells by western blot analysis. (D) Quantitative analysis of active-caspase 3 expression. LAG-3, inhibitory molecule lymphocyte activated gene-3; TIM-3, T-cell immunoglobulin and mucin domain-containing protein-3; PD-1, programmed death 1; Exo, exosome. 